\title{
Acknowledgment to Reviewers of Biologics in 2021
}

\author{
Biologics Editorial Office
}

MDPI AG, St. Alban-Anlage 66, 4052 Basel, Switzerland

Rigorous peer-reviews are the basis of high-quality academic publishing. Thanks to the great efforts of our reviewers, Biologics was able to maintain its standards for the high quality of its published papers. Thanks to the contribution of our reviewers, in 2021, the median time to first decision was 22 days and the median time to publication was 49 days. The editors would like to extend their gratitude and recognition to the following reviewers for their precious time and dedication, regardless of whether the papers they reviewed were finally published:

Adeel Malik

Agnieszka Ulatowska-Jarża

Alexis Galanis

Amulya Yaparla

Anannya Bhattacharya

Andrea Berenyiova

Andrea Caporale

Andrew Norris

Angus C. Jackson

Anna Florowska

Antimo Di Maro

Apurba Krishna Bhattacharjee

Ariane Rozza

Basanth Eedara

Citation: Biologics Editorial Office.

Acknowledgment to Reviewers of

Biologics in 2021. Biologics 2022, 2,

56-57. https://doi.org/10.3390/

biologics2010004

Published: 26 January 2022

Publisher's Note: MDPI stays neutral with regard to jurisdictional claims in published maps and institutional affiliations.

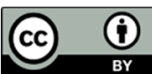

Copyright: (C) 2022 by the author. Licensee MDPI, Basel, Switzerland. This article is an open access article distributed under the terms and conditions of the Creative Commons Attribution (CC BY) license (https://creativecommons.org/license s/by/4.0/).
Beatrice Casini

Benjamin Noto

Chia-Jung Chang

Christian Setz

Corina Paul

Danuta Witkowska

Davide Bertelli

Eduardo Cilli

Elodie Monchatre-Leroy

Emilien Jeannot

Fany Reffuveille

Fernanda Rodrigues

Franciszek Główka

Gábor Ternák

Gabriele Caviglioli

Gaetano Gallo

Hongliang $\mathrm{He}$

Hyemee Kim

Hyun Jin Choi
Jaroslav Šebestík

Jerzy Juśkiewicz

Jesus Calvo-Castro

José Alberto Molina-Tijeras

José Manuel Pérez De La Lastra

Leonid Pavlovich Churilov

Marcelo Sousa Silva

Marek Kieliszek

Maria Angeles Rojo

Marieann Högman

Marika Massaro

Markus Mandl

Martin Heßling

Martin M. Edreira

Matthew Taylor

Michael Dean

Mihalj Poŝa

Minki Sung

Monika Wojciechowska

Nadia Mulinacci

Nicholas Sadgrove

Nirit Bernstein

Norbert Kiss

Olga S. Sokolova

Patrick A. Ball

Pedro Ferreira-Santos

Philippe Guerre

Remigiusz Bąchor

Robert C. Ferrier

Rubén Martín-Escolano

Sara Linden

Sena Bluemel

Sergey G. Klochkov 
Seth Pincus

Skadi Lau

Takashi Misawa
Tamer Sharafeldin

Vrba Jiri

Yoshiyuki Mishima 